\title{
Télescope
}

Revue d'analyse comparée en administration publique

\section{Les registres publics à l'ère du numérique}

\section{Olivier Glassey}

Volume 18, numéro 1-2, printemps-été 2012

Les administrations publiques à l'ère du numérique

URI : https://id.erudit.org/iderudit/1009260ar

DOI : https://doi.org/10.7202/1009260ar

Aller au sommaire du numéro

Éditeur(s)

L’Observatoire de l'administration publique

ISSN

1203-3294 (imprimé)

1929-3348 (numérique)

Découvrir la revue

Citer cet article

Glassey, O. (2012). Les registres publics à l'ère du numérique. Télescope, 18(1-2),

139-154. https://doi.org/10.7202/1009260ar
Résumé de l'article

Les registres publics contiennent des informations très riches sur les administrés. Étant de plus en plus souvent informatisés et capables d'échanger des données, ces registres pourraient constituer une ressource inestimable à l'heure de mettre en place l'administration numérique. Cela ne va par contre pas sans poser des problèmes de protection des données et implique dans tous les cas la mise en place de processus de décision quant au partage de ces données, en fonction d'objectifs précis et dans un contexte donné. Dans cet article, nous proposons un cadre d'analyse qui permet d'évaluer les besoins en information pour une prestation administrative donnée, ainsi que les possibilités de partage ou les restrictions en matière de protection de la sphère privée. 


\title{
LES REGISTRES PUBLICS À L'ÈRE DU NUMÉRIQUE
}

Par Olivier Glassey, Professeur assistant, Institut de hautes études en administration publique, Suisse • olivier.glassey@idheap.unil.ch

\begin{abstract}
RÉSUMÉ Les registres publics contiennent des informations très riches sur les administrés. Étant de plus en plus souvent informatisés et capables d'échanger des données, ces registres pourraient constituer une ressource inestimable à l'heure de mettre en place l'administration numérique. Cela ne va par contre pas sans poser des problèmes de protection des données et implique dans tous les cas la mise en place de processus de décision quant au partage de ces données, en fonction d'objectifs précis et dans un contexte donné. Dans cet article, nous proposons un cadre d'analyse qui permet d'évaluer les besoins en information pour une prestation administrative donnée, ainsi que les possibilités de partage ou les restrictions en matière de protection de la sphère privée.
\end{abstract}

\begin{abstract}
Public registers contain highly rich information about citizens. As these registers are, to a steadily increasing extent, digitized and designed for data-sharing purposes, they represent an invaluable resource when implementing e-government. At the same time, they raise a number of serious data protection issues that entail, at all events, establishing a decision-making process to frame the sharing of these data on the basis of specific objectives and in accordance with a particular context. In this article, I propose an analytical framework serving to assess both the informational requirements associated with a given administrative service as well as the opportunities for sharing data, coupled with privacy restrictions.
\end{abstract}

Pour citer cet article : Glassey, O. (2012). « Les registres publics à l'ère du numérique », Télescope, vol. 18, n 1-2, p. 139-154.

I 'administration électronique, ou l'e-government, est née avec l'essor d'Internet L dans les années 1990. L'administration en ligne ne se limite cependant pas à l'utilisation d'Internet, elle intègre également d'autres technologies de l'information et de la communication telles que la téléphonie mobile, les cartes à puce, les certificats d'identité numériques ou le réseau sans fil. Bien qu'étant un phénomène relativement jeune, l'administration électronique s'inscrit dans la lignée de l'utilisation de l'informatique et des réseaux dans les administrations publiques. Ainsi, le monde universitaire germanophone a étudié dès les années 1970 l'informatique administrative (Verwaltungsinformatik) et Bonin, dans un livre publié au début des années 1990, posait les premiers concepts théoriques de ce domaine (Bonin, 1992). Actuellement, l'étude de l'administration électronique nécessite une approche interdisciplinaire, intégrant des dimensions techniques, organisationnelles, économiques, politiques, juridiques ou sociologiques. L'objectif de cet article n'étant pas de dresser un panorama de la recherche dans le domaine, les lecteurs sont invités à se référer à des auteurs tels que Grönlund (2004), Alcaide Muñoz, Rodríguez Bolívar et López Hernánde (2010) Andersen et autres (2010) ou Fedorowicz et Dias (2010) pour en savoir plus sur ce sujet. 
En ce qui concerne les aspects pratiques de l'administration électronique, et notamment la mise en place de services en ligne, il existe deux séries d'études de référence, l'une pilotée par la Commission européenne et l'autre par les Nations Unies. Depuis 2001, la Direction générale Société de l'information et médias de la Commission européenne mandate des cabinets de conseils pour réaliser une vaste enquête sur la numérisation des services publics en Europe. La neuvième édition a été réalisée en 2010 et porte sur 32 pays, c'est-à-dire les 27 États membres plus I'Islande, la Norvège, la Suisse, la Croatie et la Turquie (Capgemini et autres, 2010). L'enquête s'intéresse principalement à la disponibilité et au niveau de sophistication de 20 prestations administratives de base, soit 12 pour les citoyens et 8 pour les entreprises (Ask, Hatakka et Grönlund, 2008), mais au fil des années de nouveaux indicateurs sont apparus, tels que l'expérience utilisateur ou l'existence d'outils "facilitateurs " (par exemple, le paiement électronique ou la gestion de l'identité numérique). En 2010, ce sont six pays qui atteignent une disponibilité en ligne de 100 \% pour les 20 prestations étudiées, avec une moyenne européenne d'un peu plus de $80 \%$. S'agissant de la sophistication, mesurée sur la base d'un modèle de maturité allant de la mise à disposition d'information jusqu'à la mise en place de services transactionnels, quatre pays atteignent les 100 \% (l'Irlande, Malte, l'Autriche et le Portugal), suivis de très près par la Suède, l'Allemagne et l'Italie (99 \%). Les écarts ne sont pas très importants, puisque la moyenne européenne s'établit à $90 \%$. Pour ce qui est de l'expérience utilisateur, qui se mesure en combinant la qualité de l'expérience interactive avec la qualité de l'information fournie, elle compte Malte, la France et les Pays-Bas dans le trio de tête.

L'enquête des Nations Unies a démarré en 2003 et classe ses 192 pays membres en fonction de l'EGDI, soit le e-government development index (Nations Unies, 2010). Ce dernier est calculé en combinant l'indice des prestations en ligne (disponibilité et maturité des prestations), l'indice de l'infrastructure de télécommunications (nombre d'ordinateurs, d'internautes, de téléphones mobiles, etc.) et l'indice du capital humain (alphabétisation et secteurs d'activité). Les pays ayant l'indice le plus élevé (0,845 et plus) sont, par ordre décroissant, la République de Corée, les États-Unis et le Canada, et les pays européens sont bien placés avec six entrées dans le top 10. Bien que la méthodologie soit critiquée, ces études comparatives ont le mérite de montrer que les prestations de base de l'administration électronique, ainsi que l'infrastructure, sont en place dans les pays développés. Cependant, les investissements massifs dans l'administration en ligne n'ont pas encore porté leurs fruits (Capgemini et autres, 2009), car les taux d'adoption ou d'utilisation des prestations en ligne restent relativement faibles. Par exemple, Malte, avec $100 \%$ de disponibilité et de sophistication des 20 prestations en ligne de base, n'atteint pas même les 50 \% de taux d'adoption par les utilisateurs (Capgemini et autres, 2009). Ce décalage est encore plus marqué pour les citoyens que pour les entreprises. En effet, selon I2010 High Level Groupe (2009), seuls 30 \% des citoyens européens ont interagi en ligne "d'une façon ou d'une autre " avec le secteur public, contre $66 \%$ pour les entreprises. La situation semble toutefois s'améliorer lentement : $42 \%$ des Européens âgés de 16 à 74 ans ont utilisé Internet pour interagir avec les administrations publiques (Capgemini et autres, 2010). En novembre 2009, la 
Déclaration ministérielle de Malmö tirait les conséquences de cet état de fait et fixait au nombre de ses priorités politiques la création des prestations qui répondent aux besoins des utilisateurs (Ministerial Declaration on e-Government, 2009). Ses auteurs insistent également sur la nécessité de la collaboration transnationale et de la réutilisation des infrastructures et des informations, en mettant l'accent sur le fait que cela ne sera possible qu'en assurant l'interopérabilité des solutions et des données. Cette déclaration ministérielle reconnaissait par ailleurs la gestion de l'identité numérique comme étant l'un des éléments facilitateurs clés pour la mise en place de l'administration électronique.

Le présent article s'inscrit dans cette logique de gestion des données et de l'identité, en s'appuyant sur le cas des registres de population pour illustrer l'approche présentée, mais en se limitant à une description de la situation européenne en la matière. La section suivante met en contexte la problématique des données des registres publics alors que la deuxième section propose un modèle conceptuel d'analyse. Enfin, la dernière section formule quelques conclusions et avance des pistes pour l'utilisation future d'un tel cadre d'analyse.

\section{- LES REGISTRES DE POPULATION}

Un registre de population est un inventaire des résidents d'un pays, avec des caractéristiques telles que la date de naissance, le sexe, l'état civil, etc., ainsi que d'autres données socioéconomiques, comme la profession ou le niveau d'éducation. Selon la Division de statistique des Nations Unies (United Nations Statistics Division, 2011), les principales fonctions administratives d'un registre de population sont de fournir des informations fiables en fonction des objectifs du gouvernement, particulièrement en termes de planification de programmes, d'établissement de budgets et de fiscalité; d'établir des numéros d'identification personnelle uniques; de vérifier l'admissibilité des personnes au droit de vote, à l'éducation, à la santé, au service militaire, aux assurances, aux services sociaux et aux systèmes de retraites; de servir de références policières et juridiques. Les registres de population sont également utiles pour estimer la population, pour planifier et évaluer le recensement de la population et pour effectuer un échantillonnage lors des enquêtes auprès des ménages. En plus de ces registres de population au sens strict, il existe une foule d'autres registres ou sources officielles de données sur les citoyens et la population, qui varient selon les pays et les contextes : registres fiscaux, fonciers, militaires, des étrangers, des poursuites et des faillites, judiciaires, de police, automobiles, etc. Des données extraites de ces registres sont utilisées dans de nombreux services administratifs, que ce soit sous la forme de certificats ou d'attestations ou via un accès direct à ces données lorsqu'elles sont numériques. Ainsi, l'admissibilité à une aide sociale ainsi que le montant de cette aide, le cas échéant, pourraient être déterminés sur la base du lieu de résidence et d'un extrait fiscal.

À l'instar des prestations administratives électroniques, de nombreux registres publics ont été numérisés ces dernières années en Europe. En parallèle à la disparition des registres papier, un phénomène de centralisation, ou du moins 
d'interopérabilité, des données est également notable. Les situations diffèrent fortement d'un pays à un autre et quelques exemples représentatifs sont donnés plus loin. Une autre tendance de fond qui influence la gestion des données publiques est la modification du cadre légal. Dans les dernières décennies, la plupart des pays européens ont adopté des lois sur la transparence et l'accès à l'information ainsi que des lois sur la protection des données. Ces lois s'appliquent généralement aux données des registres publics, mais cela implique une pesée des intérêts en matière de partage ou de protection des données. Un certain nombre de questions stratégiques sont à traiter : le partage de l'information et le respect de la sphère privée sont-ils compatibles? Faut-il mettre en place des processus de mitigation, ou d'atténuation, afin de parvenir à des compromis en fonction de contextes donnés? Ou faut-il donner la priorité à l'une ou à l'autre approche? Ces questions ne se posaient pas, ou du moins de façon beaucoup moins aiguë, lorsque les données étaient éparpillées dans des registres papier décentralisés. À l'heure où les registres sont numériques et où les échanges de données se font très facilement d'un point de vue technique, il convient de s'interroger sur la question de la gouvernance de ces données. Avant d'aller plus loin dans l'analyse théorique, la situation de cinq pays européens est présentée ci-dessous afin d'illustrer cette problématique.

La Finlande a une longue histoire en matière de registres et fut l'un des premiers pays européens à collecter des données sur sa population, dès le XVI ${ }^{\mathrm{e}}$ siècle (Population Register Centre, 2006). Des listes de recensement (un registre local de la population) ont de plus été tenues depuis 1634. Par ailleurs, la Finlande a créé un registre centralisé de la population en 1969 et un registre informatisé a été introduit en 1971, ce qui a fait de cet État un pionnier en la matière. Les citoyens finlandais ont accès aux données qui les concernent dans ce registre centralisé et ils ont le droit de demander des corrections aux autorités. Enfin, la carte d'identité électronique a été mise en place à partir de 1999 et a constitué le premier système opérationnel de ce type au monde.

Au Royaume-Uni, l'inscription des naissances, des mariages et des décès a été rendue obligatoire en $1837^{1}$. Le Royaume-Uni détient encore des registres d'état civil en format papier, complètement décentralisés; la gestion de ces registres se fait au niveau des autorités locales. De plus, les registres officiels ne sont pas accessibles au public, même s'il existe des index permettant de trouver une entrée de registre donnée. Certains de ces index locaux sont d'ailleurs publiés dans Internet et des copies certifiées des enregistrements sont délivrées pour des buts administratifs, par exemple l'obtention d'un passeport, ou généalogiques. Il n'y a aucun autre motif valable permettant d'accéder à ces registres d'état civil. Enfin, le RoyaumeUni ne possède pas de système de cartes d'identité (mis à part quelques expériences pilotes menées sur une base volontaire) et le sujet est hautement controversé dans la société britannique. Il paraît donc hautement improbable qu'une carte d'identité électronique puisse voir le jour dans les années à venir.

1 Information tirée de Wikipedia, http://en.wikipedia.org/wiki/Civil_registry. 
Le Royaume de Belgique a effectué le premier recensement de son histoire en 1946 et des registres de population détaillés sont tenus à l'échelle locale. Un registre national informatisé a fonctionné sur une base de collaboration volontaire de 1969 à 1983, année où la venue d'une nouvelle base légale a rendu cette collaboration obligatoire (Poulain, 1987). Ce registre national comprend un sous-ensemble strictement défini de données des registres locaux, ces derniers ayant toujours la responsabilité de maintenir les données. Les citoyens ne peuvent consulter ces registres locaux, mais chaque individu a le droit de demander une copie de ses propres données. En 2003, la Belgique fut également l'un des premiers pays à déployer une carte d'identité électronique (De Cock, Wouters et Preneel, 2004) et à la fin de 2009 tous les citoyens du Royaume devaient avoir une telle carte (SPF Économie, 2009).

En juin 2006, le Parlement suisse adoptait une nouvelle loi sur l'harmonisation des registres de population afin de simplifier la collecte de données statistiques et l'échange de données administratives. Par ailleurs, tous les registres d'état civil (naissances, décès, mariages et adoptions) étaient tenus dans des " grands livres " au niveau local jusqu'en 2004. Il n'y avait pas moins de 1750 registres locaux pour un pays d'une population de 7,5 millions d'habitants. L'année 2004 vit l'introduction d'une base de données fédérale et centralisée, du nom d'Infostar (Confédération suisse, 2003). En plus de ces registres d'état civil, la Suisse compte encore environ 2500 registres de résidence, appelés " Contrôle de l'habitant " et, à quelques exceptions près, maintenus au niveau communal. Les citoyens et les résidents suisses sont souvent appelés à demander des extraits d'état civil ou des attestations de résidence, lesquels constituent un préalable à l'accomplissement de nombreux services administratifs. En 2009, le peuple suisse a accepté à une très petite majorité $(50,1 \%)$ l'introduction d'un nouveau passeport biométrique (TSR.ch, 2010) et l'un des sujets les plus brûlants de la campagne qui a précédé la votation fut la possible utilisation d'une puce électronique sur la future carte d'identité électronique.

En Roumanie, le premier recensement a été réalisé en 1838 (Galceava, Chiran et Dragusin, 2010). Actuellement, les registres de population sont gérés au niveau local, sous la houlette du ministère de l'Administration et de l'Intérieur, par l'intermédiaire de l'Inspectorat national des registres de population, ce dernier étant également responsable de la délivrance des cartes d'identité. Il existe par ailleurs un numéro d'identification personnel qui est généré et administré par le Centre national de gestion des bases de données de la population ${ }^{2}$. Les citoyens ont un accès limité à leurs données, soumis à un processus administratif d'autorisation relativement laborieux. En juillet 2010, le registre national roumain de la population a été centralisé et un projet pilote de délivrance de cartes d'identité numérique était prévu pour 2011.

Ces quelques exemples montrent bien la diversité de la situation en matière de registres de population (complètement centralisés ou décentralisés, décentralisés

2 Information tirée de Wikipedia, http://en.wikipedia.org/wiki/National_identification_number. 
avec agrégation de données au niveau central, numérisés ou non, etc.) de même que les problématiques d'accès (complet, limité, sur demande expresse ou de routine). Ils illustrent également les différences culturelles en matière d'identité : en Finlande le numéro d'identification personnel unique est couramment utilisé et échangé, alors qu'au Royaume-Uni c'est l'existence même d'une carte d'identité qui n'est pas acquise. Ces différences s'observeront également plus loin dans l'article lorsque les thèmes de la transparence ou de la protection des données seront abordés. Ces pratiques diverses ont des répercussions sur l'administration publique, notamment en matière de provision de services électroniques, et plus généralement de management public. Le discours présentant le programme du nouveau gouvernement du canton de Genève en 2005 en constitue un bon exemple : dans la section consacrée à l'administration en ligne, il est dit : " [U]n citoyen, un résident, une entreprise ne devrait transmettre qu'une seule fois aux autorités les informations dont elles ont besoin, par internet pour ceux qui le souhaitent " (Secrétariat du Grand Conseil, p. 11). Cela signifierait concrètement que dès lors qu'une information est dans un registre de population, l'administration ne devrait plus avoir à la demander et elle devrait pouvoir y accéder directement afin de mener à bien la procédure administrative demandée. La pratique est encore très éloignée de ce discours d'intention et ne va pas sans poser de problèmes juridiques, organisationnels, voire sociologiques. Pour conclure dans une perspective de management public, reprenons les termes de Pollitt (2009) qui indique que les registres de population ont suscité très peu d'attention du monde académique jusqu'à présent, que ce soit sous la forme de projets de recherche ou de publications scientifiques. En effet, ces registres ont été étudiés largement d'un point de vue historique, généalogique, statistique ou démographique, mais très peu, voire pas du tout, dans le domaine de l'administration ou du management publics.

\section{- LE MODÈLE CONCEPTUEL D'ANALYSE}

L'objectif étant d'étudier l'utilisation des données des registres publics dans le cadre de la réalisation de services administratifs, il convient de développer un modèle d'analyse couvrant ces deux dimensions. Le tableau 1 présente les dimensions d'analyse des prestations administratives et le tableau 2 reprend les dimensions portant sur la gouvernance et le partage des données publiques. Chacune des dimensions est également brièvement discutée.

\section{L'analyse des prestations administratives}

Les deux dimensions d'analyse présentées ci-dessous sont adaptées d'un modèle développé pour analyser plus globalement l'administration en ligne (Glassey, 2012). 


\section{TABLEAU 1 : LES DIMENSIONS D'ANALYSE DES PRESTATIONS ADMINISTRATIVES}

\begin{tabular}{|c|c|}
\hline OFFRE & DEMANDE \\
\hline $\begin{array}{l}\text { - Disponibilité } \\
\text { - Sophistication }\end{array}$ & $\begin{array}{l}\text { - Adoption } \\
\text { - Usage } \\
\text { - Expérience utilisateur }\end{array}$ \\
\hline
\end{tabular}

\section{L'offre}

La " disponibilité " indique simplement si une prestation administrative est disponible sous sa forme électronique. Le concept de "sophistication " (ou de maturité) de l'administration électronique repose sur un découpage en niveaux de maturité de l'administration en ligne, variant généralement de trois à six (Glassey, 2002). Une tendance se dégage toutefois autour des quatre niveaux de sophistication suivants :

- la mise à disposition d'information : il s'agit typiquement d'un portail où l'information est organisée de façon thématique (par exemple autour des événements de la vie courante ou de situations auxquelles une entreprise pourrait être confrontée) et les utilisateurs y accèdent de façon transparente, sans avoir à se soucier de la hiérarchie ou de l'organisation interne du secteur public. Cette catégorie recouvre également des outils plus avancés qui permettent d'effectuer des requêtes dans des bases de données officielles, par exemple le registre du commerce;

- les services électroniques transactionnels : la prestation administrative peut être accomplie de bout en bout de façon électronique, comme c'est le cas avec la déclaration de revenus en ligne;

- l'intégration de processus métiers ou de procédures : une procédure administrative répartie entre plusieurs offices ou départements peut être réalisée de façon intégrée, sans avoir à faire le tour des offices concernés. Un exemple classique en est la demande d'autorisation de construire, où de nombreuses parties prenantes peuvent être amenées à donner un préavis. Un processus intégré de gestion de flux de travail permet ainsi à l'utilisateur de déposer une demande d'autorisation de construire et d'en suivre la progression de façon transparente, c'est-à-dire en masquant la complexité des procédures administratives sous-jacentes;

- la transformation des métiers : il ne s'agit là plus uniquement d'intégrer des procédures existantes, mais d'utiliser le levier des technologies de l'information et de la communication pour réorganiser le fonctionnement de l'administration. L'introduction en Suisse du registre informatisé de l'état civil, Infostar, décrit à la section précédente, a été menée en parallèle à une réduction du nombre d'offices d'état civil d'un facteur 10. La numérisation de ces registres n'a pas été l'unique cause de cette rationalisation, mais elle en a été un élément facilitateur. 


\section{La demande}

L' " adoption " par les utilisateurs est un facteur bien documenté. Par exemple, Eurostat (2010) a publié les mesures d'utilisation d'Internet par les individus et les entreprises pour interagir avec les administrations publiques. Une autre dimension proposée dans ce modèle repose sur la catégorisation des « usages " présentée par Wimmer et Tambouris (2002), décrite et illustrée par des exemples ci-dessous :

- la récolte d'information et la formation des intentions d'usage : par exemple un citoyen se renseigne sur la possibilité de demander une aide financière pour son logement et utilise une calculatrice en ligne pour déterminer s'il est admissible (Atkinson, Bench-Capon et McBurney, 2004);

- la demande de prestation : il existe de nombreux exemples de demandes de prestation, tels que la demande de certificats ou d'attestations ou la prise de rendez-vous;

- la réception ou la réalisation d'une prestation : cette étape est encore très fréquemment effectuée sous format papier, puisque c'est là le seul format officiellement reconnu pour nombre d'autorisations, de certificats ou de documents. Seules les prestations les plus simples peuvent être effectuées entièrement en ligne, par exemple la prise de rendez-vous pour le contrôle technique d'un véhicule ou la demande d'un délai pour déposer sa déclaration de revenus;

- le suivi : cette phase regroupe le traitement des réclamations et des recours, les demandes d'explications complémentaires, les vérifications, l'évaluation de la satisfaction des utilisateurs, etc.

Dans son enquête sur le développement de l'administration électronique, la Commission européenne indique que l' "expérience utilisateur " est désormais reconnue comme un élément de mesure essentiel de l'adoption des prestations en ligne, tout en concédant que c'est un domaine complexe à analyser car la culture et les normes y jouent un rôle majeur (Capgemini et autres, 2009). À cet effet, Kubicek et Noack (2010) ont comparé l'introduction de systèmes de gestion de l'identité dans huit pays européens. Ils ont constaté que ces systèmes sont plus largement utilisés dans les pays où il y a moins de barrières à l'entrée (coûts, installation de logiciels, acquisition de matériel tel qu'une clé USB, etc.), même si ces mesures ont pour but d'offrir une meilleure sécurité. À l'inverse, les pays qui ont exigé des méthodes d'authentification forte n'ont pas eu le succès escompté, très certainement à cause de la complexité technique additionnelle imposée aux utilisateurs. L'expérience utilisateur peut se mesurer de façon simple (et certainement limitative) sur la base de deux éléments définis par la Commission européenne (Capgemini et autres, 2010) :

- la qualité de l'interaction : comment les utilisateurs perçoivent-ils la facilité d'utiliser une prestation en ligne?

- la qualité de l'information : quelle est la valeur ajoutée de la prestation en ligne en termes d'information pertinente pour les utilisateurs?

Ces deux dimensions s'inspirent du modèle d'acceptation de la technologie (Davis, 1989), qui part de l'hypothèse que lorsque des utilisateurs sont confrontés à une nouvelle technologie, de nombreux facteurs influencent l'utilisation qu'ils en 
feront, en particulier la perception de la facilité de l'utilisation et la perception de l'utilité de cette technologie. Cette approche est ici adaptée au cas particulier des prestations de l'administration électronique.

\section{L'analyse des données publiques}

Afin d'analyser plus spécialement la gestion des données des registres publics, ainsi que la possibilité de les partager ou non, nous intégrons ici deux dimensions d'analyse pour définir les métadonnées liées aux registres de population (Glassey, 2011) : la gouvernance et le partage des données. Avant d'aller plus loin, il est nécessaire de définir brièvement la gouvernance des données. Le terme " gouvernance " est très largement utilisé sans qu'il y ait forcément de consensus quant à sa signification précise. Cette notion est toutefois relativement bien formalisée dans deux domaines : la gouvernance politique (mondiale, locale, territoriale, etc.) et la gouvernance d'entreprise. Ces dernières partagent un certain nombre d'attributs : des objectifs à atteindre, des processus de décision et l'implication des parties prenantes, qu'il s'agisse des actionnaires, d'un conseil d'administration, de politiciens, de citoyens ou de groupes d'intérêts particuliers. Nous transposons donc ces attributs aux données des registres publics, en nous intéressant aux objectifs à atteindre (protection des données, partage ou mitigation), aux processus de décision et aux parties prenantes.

\section{TABLEAU 2 : LES DIMENSIONS D'ANALYSE DES DONNÉES PUBLIQUES}

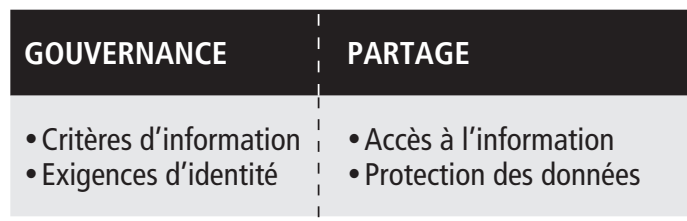

\section{La gouvernance des données}

Les " critères d'information " définis par CobiT (IT Governance Institute, 2007) sont utilisés dans notre modèle afin d'analyser et de qualifier les données des registres. CobiT est un cadre de référence dans le domaine de la gouvernance des technologies de l'information qui propose un ensemble de bonnes pratiques afin de valider l'alignement des systèmes d'information avec les processus métiers et la stratégie d'une organisation. Pour l'analyse de la dimension "information ", CobiT s'appuie sur les critères suivants :

- l'efficacité : l'information est pertinente, correcte, consistante, utilisable et disponible en temps voulu;

- l'efficience : l'information est fournie en utilisant les ressources disponibles de façon optimale;

- la confidentialité : l'information sensible n'est pas divulguée de façon non autorisée; 
- l'intégrité : l'information est exacte, valide et complète;

- la disponibilité : l'information est disponible en fonction des besoins;

- la conformité : l'information est utilisée conformément au cadre légal et aux règlements internes;

- la fiabilité : l'information est digne de confiance.

Tous ces critères ne doivent évidemment pas être respectés dans tous les contextes : il s'agit d'un cadre d'analyse qui permet de qualifier les données. CobiT propose simplement de qualifier ces critères comme étant "primaires ", " secondaires " ou " non applicables ", mais il est certes possible d'établir ses propres échelles de qualification. Ainsi, le niveau de confidentialité pourrait être défini comme " public ", " accès restreint " ou " confidentiel ".

En ce qui concerne les critères de qualité, de fiabilité et d'intégrité de l'information, il convient de mentionner qu'il existe des approches permettant d'analyser et de valider de façon partiellement automatique les données des registres de population. Ce n'est par contre pas l'objet de cet article, les lecteurs désirant des explications complémentaires sur ces problématiques peuvent consulter entre autres Boydens (2011) ou Olson (2003). Un cas particulier de la gestion de la qualité et de la fiabilité des données des registres publics est celui de l'identification des adresses (postales ou spatiales), qui est traité dans le cadre de recherches telles que le projet européen Inspire ${ }^{3}$.

Une autre dimension également primordiale pour la gestion des données des registres publics est celle de la pérennité et de la préservation à moyen et long terme de l'information numérique (voir Olson, 2009), mais cet aspect n'est pas non plus traité dans cet article, qui se concentre sur des aspects opérationnels de la gouvernance des données plutôt que sur la gestion patrimoniale des informations.

Nous n'avons pas encore abordé dans le détail la question des " exigences " en matière de " gestion de l'identité ", mais elle est centrale pour effectuer un travail d'analyse quant à l'accès aux données des registres publics. Les administrés ont des identités multiples lorsqu'ils sont en relation avec le secteur public : ils peuvent être contribuables, parents, membres d'un ménage, soldats, assurés, voire inculpés ou prisonniers. À cet effet, Lips, Taylor et Organ (2006) affirment que l'identification personnelle est indispensable à la provision de nombreuses prestations administratives. Ils ajoutent qu'historiquement et de façon typique, ces processus d'identification sont basés sur une approche manuelle et personnelle : un administré tend sa carte d'identité à un fonctionnaire qui valide le formulaire papier soumis par l'administré. Ainsi, pour chaque procédure administrative un formulaire est saisi puis archivé (et potentiellement oublié à tout jamais). Cela contraste singulièrement avec l'approche décrite à la section précédente où les administrés ne devraient transmettre qu'une seule fois à l'administration les renseignements dont celle-ci a besoin pour accomplir une procédure administrative.

Pour permettre l'accès à des données numérisées, disponibles dans les registres de population ou dans d'autres bases de données, des mécanismes de gestion

3 Voir http://inspire.jrc.ec.europa.eu/. 
de l'identité et de délégation sont absolument indispensables. Un citoyen pourrait ainsi s'identifier et autoriser l'accès à ses données à une unité administrative pour un temps donné et dans un but précis, cette dernière devant évidemment s'identifier à son tour pour y accéder. Précisons encore qu'il ne s'agit ici nullement de questions d'infrastructures techniques de gestion d'identité, mais de processus de gestion. Afin d'analyser ces derniers, nous utilisons les « lois de l'identité » décrites par Cameron (2005). Celui-ci définit l'identité numérique comme un ensemble de déclarations effectuées par un "sujet numérique " à propos de lui-même ou d'autres sujets ou objets numériques. Par exemple, ces déclarations peuvent être : " Je m'appelle Paul et j'ai plus de 18 ans ", " Je m'appelle Paul et je suis marié à Virginie " ou " Je m'appelle Paul, j'ai deux enfants et un revenu qui m'autorise à demander une aide sociale. " Afin de gérer l'identité numérique en fonction de ces déclarations, Cameron (2005) définit sept " lois de l'identité » :

- le consentement et le contrôle par l'utilisateur : un système de gestion de l'identité numérique ne doit révéler de l'information d'identification qu'avec le consentement de l'utilisateur et sous son contrôle;

- la divulgation minimale : le système doit divulguer le strict minimum nécessaire en termes d'information d'identification, un système limitant l'utilisation de cette information est le plus stable sur le long terme;

- la justification des parties tierces : les systèmes de gestion d'identité doivent être conçus de façon à ce que la diffusion d'information d'identification soit limitée aux parties tierces qui peuvent justifier de la nécessité d'intervenir dans une relation d'identité;

- l'identité dirigée : un système de gestion d'identité doit supporter tant les identifiants "bidirectionnels " pour l'utilisation par le secteur public que les identifiants " unidirectionnels " pour les entités privées afin de faciliter l'identification tout en empêchant de mettre en correspondance des données concernant le sujet identifié. Ainsi une approche de " transcodage " devrait permettre d'identifier un administré comme ayant droit à une aide sociale sans toutefois permettre de savoir qui sont les administrés qui perçoivent une telle aide;

- le pluralisme des opérateurs : les systèmes de gestion d'identité doivent être interopérables et compatibles techniquement afin de pouvoir utiliser les services de multiples fournisseurs de services d'identité;

- l'intégration humaine : un système de gestion d'identité doit considérer l'utilisateur comme un " composant " et mettre à sa disposition des outils de communication protégés et non ambigus;

- une expérience utilisateur cohérente au-delà des frontières du système : les systèmes de gestion d'identité doivent offrir une expérience utilisateur simple et cohérente, tout en permettant la séparation des contextes en fonction des opérateurs et des technologies.

Selon Cameron (2005), ces lois devraient permettre aux utilisateurs de se sentir sûrs et protégés lorsqu'ils effectuent des transactions en ligne. Elles sont plus de l'ordre des bonnes pratiques que de celui d'indicateurs opérationnalisables, mais nous considérons qu'une évaluation de ces dimensions en termes de maturité 
répond à nos besoins d'analyse. L'approche par modèle de maturité est relativement courante et elle est notamment détaillée dans le rapport de l'IT Governance Institute (2007). Pour conclure cette partie sur la gestion de l'identité, mentionnons que d'autres préalables sont indispensables à la mise en place de tels systèmes : l'authentification, la confiance, l'imputabilité, l'ergonomie, la gestion du cycle de vie des déclarations d'identité (Casassa Mont et autres, 2002), ainsi que la localisation (les identités sont liées à un contexte donné et un individu peut avoir des identités différentes en fonction du contexte), la réciprocité ou les principes de compréhension mutuelle (Roussos, Peterson et Patel, 2003). Ces préalables ne sont toutefois pas intégrés dans notre modèle d'analyse, car ils ne sont pas à proprement parler des mécanismes de gestion ou de gouvernance.

\section{Le partage des données}

Le partage des connaissances est une thématique qui est étudiée dans le domaine de la gestion des connaissances, où Pardo et ses collègues (2006) notent que la recherche porte sur les formes de connaissances à partager (tacites/explicites) ainsi que sur les processus et la technologie qui permettent de partager des connaissances. Nous appliquons ces idées à un sous-ensemble de connaissances explicites, soit les informations contenues dans les registres publics, en considérant que la source du partage est le secteur public et les destinataires en sont les administrés qui accèdent à ces informations. Cet accès est de plus garanti juridiquement dans de nombreux pays, puisqu'en 2006 il y avait déjà 70 États qui avaient passé des lois sur l'accès à l'information ou sur la transparence des données publiques (Banisar, 2006). L'existence d'un cadre légal n'assure cependant pas automatiquement le partage d'information : une enquête réalisée dans 14 pays en 2006 a montré que seules $33 \%$ des demandes d'information ont été satisfaites et que $38 \%$ n'ont jamais reçu de réponses (Open Society Justice Initiative, 2006). Par ailleurs, il s'agissait là de partager l'information de manière réactive (en répondant à une demande spécifique) et non proactive (en mettant à disposition de l'information existante). Cela s'explique notamment par le fait que le partage d'information ne fait pas partie de la culture de la plupart des administrations publiques, qui sont encore loin de mettre à disposition systématiquement l'information dont elles disposent. L'accès à l'information se mesure en répertoriant les données qui devraient être accessibles selon la loi et en identifiant celles qui sont réellement accessibles, en fonction de quelles règles d'accès (par exemple librement accessibles sur Internet ou disponibles uniquement sur demande motivée).

De nombreux pays ont d'autre part une législation qui porte sur la protection de la sphère privée ou sur la protection des données personnelles. Pour une discussion sur les concepts de la sphère privée et l'historique de la protection des données, les lecteurs peuvent se référer à Glassey (2011). Pour définir des indicateurs portant sur la protection des données, nous utilisons les travaux de Otjacques, Hitzelberger et Feltz (2006) qui ont effectué une enquête sur l'implémentation de la directive 95/46/EC de la Commission européenne sur la protection des données. Elle portait notamment sur (1) l'obligation de notifier une autorité de contrôle lorsque des données personnelles sont traitées en masse, ainsi que les catégories 
d'exception spécifiquement prévues dans la loi; (2) les droits des individus d'être informés lorsqu'une autorité utilise les données les concernant; (3) les droits des individus d'accéder librement à leurs propres données; (4) le partage des données entre les administrations sur la base d'un identifiant unique, avec ou sans autorité de contrôle.

Ces critères sont appliqués de façon binaire, en indiquant simplement s'ils existent ou non.

\section{- CONCLUSIONS}

Dans cet article nous avons en premier lieu voulu montrer l'importance des données de registres publics pour l'administration à l'ère du numérique. En effet, la qualité des services offerts aux administrés pourrait être grandement améliorée en utilisant directement l'information présente dans les différents registres de population, d'état civil, de résidence, fiscaux ou autres. De nombreux services administratifs qui n'en sont pas réellement, du moins du point de vue des citoyens (demandes d'attestation ou d'extraits de registre), pourraient même être appelés à disparaître à terme. Évidemment, un partage généralisé de l'information n'irait pas sans poser des problèmes juridiques, puisque la protection des données privées est garantie dans plusieurs pays. Afin de mieux saisir les enjeux liés à un contexte donné, dont quelques exemples européens ont été donnés dans cet article, nous proposons un cadre d'analyse qui a pour but de combiner les aspects de prestations administratives d'une part, et ceux de gouvernance des données d'autre part. Ainsi, pour une prestation donnée, ce cadre devrait permettre d'analyser les différentes dimensions de partage ou de protection des données qui y sont liées.

En ce qui concerne la validation de ce cadre d'analyse, elle n'a pas encore été effectuée de façon empirique pour la globalité des dimensions. La partie sur l'analyse des prestations administratives a été appliquée au domaine de la taxation électronique (Glassey, 2010) et la partie portant sur la gouvernance des données est actuellement utilisée dans le cadre d'un travail de thèse (Formaz et Glassey, 2010). Séparément, les deux parties du modèle d'analyse fonctionnent relativement bien, mais leur intégration reste à tester. Nous travaillons actuellement sur la mise en place d'un projet de comparaison entre deux pays européens, qui permettrait d'affiner et de valider ce modèle intégré. 


\section{BIBLIOGRAPHIE}

Alcaide Muñoz, L., M. P. Rodríguez Bolívar et A. M. López Hernánde (2010). Analyzing the e-Government Research (2000-2009): State of the Art, Contextualization and Research Opportunities, Communication présentée au 4th International Conference on Methodologies, Technologies and Tools Enabling e-Government, 1-2 juillet, Olten, Suisse.

Andersen, K. N. et autres (2010). " Fads and Facts of e-Government: A Review of Impacts of e-Government (2003-2009) ", International Journal of Public Administration, vol. 33, $\mathrm{n}^{\circ} 11$, p. $564-579$.

Ask, A., M. Hatakka et $\AA$. Grönlund (2008). " The Örebro City Citizen-Oriented e-Government Strategy ", International Journal of Electronic Government Research, vol. 4, $\mathrm{n}^{\circ}$ 4, p. 69-88.

Atkinson, K., T. Bench-Capon et P. McBurney (2004). " PARMENIDES: Facilitating Democratic Debate ", dans R. Traunmüller (dir.), Electronic Government: Third International Conference, EGOV 2004, Berlin, Springer, p. 313-316.

Banisar, D. (2006). Freedom of Information around the World 2006: A Global Survey of Access to Government Information Laws, Privacy International.

Bonin, H. E.G. (dir.) (1992). Verwaltungsinformatik - Konturen einer Disziplin, Mannheim, BI Wissenschaftsverlag.

Boydens, I. (2011). «Strategic Issues Relating to Data Quality for e-Government: Learning from an Approach Adopted in Belgium ", dans S. Assar, I. Boughzala et I. Boydens (dir.), Practical Studies in e-Government: Best Practices from Around the World, New York, Springer p. 113-130.

Cameron, K. (2005). The Laws of Identity, www.identityblog.com/stories/2005/05/13/ TheLawsOfIdentity.pdf (page consultée le 21 décembre 2011).

Capgemini et autres (2010). Digitizing Public Services in Europe: Putting Ambition into Action, www.ec.europa.eu/information_society/newsroom/cf/document.cfm?action= display\&doc_id=747 (page consultée le 28 juin 2011).

Capgemini et autres (2009). Smarter, Faster, Better e-Government, www.ec.europa.eu/ information_society/eeurope/i2010/docs/benchmarking/egov_benchmark_2009. pdf (page consultée le 28 juin 2011).

Casassa Mont, M. et autres (2002). Identity Management: A Key e-Business Enabler, HP Laboratories Bristol.

Confédération suisse (2003). Introduction progressive d'Infostar, www.bj.admin.ch/bj/fr/ home/dokumentation/medieninformationen/2003/2003-03-13.html (page consultée le 21 décembre 2011).

Davis, F. D. (1989). « Perceived Usefulness, Perceived Ease of Use, and User Acceptance of Information Technology ", MIS Quarterly, vol. 13, n³, p. 319-340.

De Cock, D., K. Wouters et B. Preneel (2004). «Introduction to the Belgian EID Card BELPIC ", Proceedings of EuroPKI 2004, p. 1-13

Eurostat (2010). E-government Statistics, http://epp.eurostat.ec.europa.eu/statistics_ explained/index.php/E-government_statistics - Use_of_online_services_by_the_ citizens (page consultée le 28 juin 2011). 
Fedorowicz, J. et M. A. Dias (2010). " A Decade of Design in Digital Government Research ", Government Information Quarterly, vol. 27, n 1, p. 1-8.

Formaz, J. et O. Glassey (2010). " A Three-Dimensional Framework to Analyse the Governance of Population Registers ", dans M. Bezzi et autres (dir.), Privacy and Identity Management for Life, Springer, p. 112-121.

Galceava, I. B., C. V. Chiran et M. Dragusin (2010). "Statistics in Romania: From Catagraphies to Census - Past and Reality ", Revista Romana Statistica, vol. 58, n 10, p. $49-57$.

Glassey, O. (2012). "L'administration en ligne : quand les utilisateurs deviennent des agents publics ", Manuel suisse d'administration publique, Lausanne, PPUR.

Glassey, O. (2011). " Metadata for Identity Management of Population Registers ", Future Internet, vol. 3, n 2 , p. 130-143.

Glassey, O. (2010). Why TaxMe Makes Taxpayers Happy, Communication présentée au 10th European Conference on E-government, 17-18 juin, University of Limerick, Ireland.

Glassey, O. (2002). Modélisation et implémentation d'un guichet virtuel pour les administrations publiques, http://doc.rero.ch/lm.php?url=1000,40,5,20050413103236-ST/1_These_ Glassey.pdf (page consultée le 28 juin 2011).

Grönlund, Å. (2004). "State of the Art in e-Gov Research: A Survey », dans R. Traunmüller (dir.), Electronic Government: Third International Conference, EGOV 2004, Berlin, Springer, p. 178-185.

I2010 High Level Groupe (2009). Benchmarking Digital Europe 2011-2015: A Conceptual Framework, http://ec.europa.eu/information_society/eeurope/i2010/docs/benchmarking/ benchmarking_digital_europe_2011-2015.pdf (page consultée le 28 juin 2011).

IT Governance Institute (2007). CobiT 4.1, Rolling Meadows, IT Governance Institute.

Kubicek, H. et T. Noack (2010). " Different Countries-different Paths Extended Comparison of the Introduction of eIDs in Eight European Countries ", Identity in the Information Society, vol. 3, n 1, p. 235-245.

Lips, A. M. B., J. A. Taylor et J. Organ (2006). « Identity Management as Public Innovation: Looking Beyond ID Cards and Authentication Systems ", dans V. J. J. M. Bekkers, H. P. M. van Duivenboden et M. Thaens (dir.), ICT and Public Innovation: Assessing the Modernisation of Public Administration, Amsterdam, IOS Press, p. 204-216.

Ministerial Declaration on eGovernment (2009). www.egov2009.se/wp-content/uploads/ Ministerial-Declaration-on-eGovernment.pdf (page consultée le 21 décembre 2011).

Nations Unies (2010). E-Government Survey 2010, http://unpan1.un.org/intradoc/groups/ public/documents/un/unpan038851.pdf (page consultée le 28 juin 2011).

Olson, J. (2009). Database Archiving, MK - OMG Press.

Olson, J. (2003). Data Quality: The Accuracy Dimension, Elsevier, The Morgan-Kaufmann Series in Database Management.

Open Society Justice Initiative (2006). Transparency and Silence: A Survey of Access to Information Laws and Practices in 14 Countries, New York, Open Society Justice Initiative.

Otjacques, B., P. Hitzelberger et F. Feltz (2006). « Identity Management and Data Sharing in the European Union ", dans Proceedings of the 39th Annual Hawaii International Conference on System Sciences (HICSS'06) - Track 6, Kauai, Computer Societry Press. 
Pardo, T. A. et autres (2006). "Knowledge Sharing in Cross-boundary Information System Development in the Public Sector ", Information Technology and Management, vol. $7, n^{\circ} 4$, p. 293-313.

Pollitt, C. (2009). Backwater? Conditions for Hyper-Stability in an Age of Hyper-Innovation, Commincation présentée au 13th IRSPM Conference, 6 au 8 avril, Copenhagen.

Population Register Centre (2006). History, www.vrk.fi/default.aspx?id=42 (page consultée le 21 décembre 2011).

Poulain, M. (1987). "The Measurement of International Migration in Belgium ", International Migration Review, vol. 21, n 4, p. 1107-1137.

Roussos, G., D. Peterson et U. Patel (2003). « Mobile Identity Management: An Enacted View ", International Journal of Electronic Commerce, vol. 8, n 1, p. 81-100.

Secrétariat du Grand Conseil (2007). Projet de loi pour le développement de l'administration en ligne, www.ge.ch/grandconseil/data/texte/PL10177.pdf (page consultée le 21 décembre 2011).

SPF Économie (2009). La carte d'identité électronique, www.economie.fgov.be/fr/ consommateurs/Internet/e_Government/e_ID/index.jsp (page consultée le 21 décembre 2011).

TSR.CH (2010). Passeport biométrique : oui de justesse, www.tsr.ch/info/suisse/1040015passeport-biometrique-oui-de-justesse.html (page consultée le 21 décembre 2011).

United Nations Statistics Division (2011). Population Registers, http://unstats.un.org/unsd/ demographic/sources/popreg/popregmethods.htm (page consultée le 21 décembre 2011).

Wikipedia (2011a). Civil Registry in England and Wales, http://en.wikipedia.org/wiki/Civil_ registry (page consultée le 21 décembre 2011).

Wikipedia (2011b). National Identification Number: Romania, http://en.wikipedia.org/wiki/ National_identification_number (page consultée le 21 décembre 2011).

Wimmer, M. et E. Tambouris (2002). Online One-Stop Government: A Working Framework and Requirement, Communication présentée au IFIP World Computer Congress, 26 au 30 août, Montréal. 